\title{
The Rostromedial Tegmental Nucleus: Anatomical Studies and Roles in Sleep and Substance Addictions in Rats and Mice
}

This article was published in the following Dove Press journal: Nature and Science of Sleep

\author{
Ya-Nan Zhao (1) ${ }^{1, *}$ \\ Yu-Dong Yan ${ }^{1} *$ \\ Chen-Yao Wang (D) \\ Wei-Min Qu (iD) \\ Thomas C Jhou ${ }^{2}$ \\ Zhi-Li Huang $\mathbb{D}^{\prime}$ \\ Su-Rong Yang (D)
}

'Department of Pharmacology, School of Basic Medical Sciences, Fudan University, Shanghai, People's Republic of China; ${ }^{2}$ Department of Neuroscience, Medical University of South Carolina, Charleston, SC, USA

*These authors contributed equally to this work
Correspondence: Su-Rong Yang;

Zhi-Li Huang

Department of Pharmacology, School of Basic Medical Sciences, Fudan University, Shanghai, People's Republic of China

$\mathrm{Tel} / \mathrm{Fax}+86-2 \mathrm{I}-54237228$;

+86-21-54237I03

Email sryang@shmu.edu.cn;

huangzl@fudan.edu.cn

\begin{abstract}
The rostromedial tegmental nucleus (RMTg), a brake of the dopamine system, is specifically activated by aversive stimuli, such as foot shock. It is principally composed of gamma-aminobutyric acid neurons. However, there is no exact location of the RMTg on the brain stereotaxic atlas. The RMTg can be defined by c-Fos staining elicited by psychostimulants, the position of retrograde-labeled neurons stained by injections into the ventral tegmental area (VTA), the terminal field formed by axons from the lateral habenula, and some molecular markers identified as specifically expressed in the RMTg such as FoxP1. The RMTg receives a broad range of inputs and produces diverse outputs, which indicates that the RMTg has multiple functions. First, the RMTg plays an essential role for non-rapid eye movement sleep. Additionally, the RMTg serves a vital role in response to addiction. Opiates increase the firing rates of dopaminergic neurons in the VTA by acting on $\mu$-opioid receptors on RMTg neurons and their terminals inside the VTA. In this review, we summarize the recent research advances on the anatomical location of the RMTg in rats and mice, its projections, and its regulation of sleep-wake behavior and addiction.
\end{abstract}

Keywords: rostromedial tegmental nucleus, anatomical location, projections, sleep-wake behavior, addiction

\section{Introduction}

The ventral tegmental area (VTA) is well known as an important midbrain dopaminergic (DAergic) nucleus. In addition to DAergic neurons, gamma aminobutyric acid (GABA) ergic neurons are also an important type of neurons in the VTA. ${ }^{1}$ GABAergic VTA neurons communicate locally within the VTA but also give rise to prominent projections to other parts of the brain ${ }^{2,3}$ and regulate sleep, mania-like behavior, and innate defensive responses. ${ }^{4-6}$ In 2005, Perrotti et al found that chronic administration of psychostimulants, such as cocaine and amphetamine, to rats could induce the expression of the transcription factor Delta FosB within a group of GABAergic neurons in the posterior VTA, but no expression was observed in the anterior VTA, the traditional VTA area. ${ }^{7}$ Jhou et al also reported GABAergic neurons lateral to the median raphe nucleus and caudal to the VTA regulate freezing and other passive aversive responses via projections to midbrain DAergic neurons. ${ }^{8}$ At the beginning of 2009 , Kaufling et al first proposed the term "tail of the VTA". In the same year, Jhou et al published two papers on the same structure, which introduced the term "RMTg". 10,11 There is now consensus that the RMTg is a distinct mesopontine GABAergic structure that stretches from the caudal pole of the VTA deep into the mesopontine tegmentum and that the RMTg and the tail of the VTA 
both refer to the same structure. Later, researchers began to further investigate the roles and neural circuits of the RMTg in neurobiology. In 2018, we found a novel role for the RMTg regulating sleep-wake behavior. ${ }^{12}$ These results contribute to the emerging recognition of the RMTg's importance in multiple brain functions.

\section{Anatomical Location for the Delimitation of the RMTg} Anatomical Location for RMTg Delimitation in Rats by Observing c-Fos Expression in Neurons

The RMTg, rich in $\mu$-opioid receptors, is also immunoreactive for somatostatin and expresses glutamic acid decarboxylase (GAD) 67 mRNA. This nucleus is located dorsolateral to the caudal part of the interpeduncular nucleus (IPN) and has outstandingly dense efferent projections to the VTA and compact part of the substantia nigra (SNc). ${ }^{11,13,14}$ When rats are administered psychostimulants, such as modafinil, ${ }^{15}$ D-amphetamine, ${ }^{11,16}$ or cocaine, ${ }^{17}$ a large number of neurons in the RMTg are induced to express immunoreactivity against the immediate-early gene product c-Fos.

Similarly, shock or shock-predictive sound cues could also activate RMTg neurons. ${ }^{8}$ Therefore, the anatomical location of the RMTg is usually defined by injecting the retrograde tracer of cholera toxin B subunit (CTB) into the VTA and then observing the co-expression of c-Fos and CTB-labeled neurons in the RMTg area. ${ }^{10}$ There is partial intermingling of the most posterior tyrosine hydroxylase (TH)-positive neurons at the VTA with the most rostral extent of the RMTg, but the middle and posterior levels of the RMTg show almost no mingling with TH-positive neurons. According to brain slice staining and comparison with the atlas, the anatomical location of the RMTg in rats is from $6.2 \mathrm{~mm}$ to $8.8 \mathrm{~mm}$ posterior to the bregma.

\section{Anatomical Location for RMTg Delimitation in Mice by Nerve Fiber Projections}

The anatomical position of the RMTg was first defined in rats, and a series of studies on its projections and functions were carried out mainly in rats. However, the emergence and use of transgenic mice, combined with optogenetics and chemogenetics, have made it possible to manipulate the activities of specific types of neurons. Therefore, determining the anatomical location of the RMTg in mice is vital to explore its neurological functions and specific neural circuits.
Given that the RMTg is strongly innervated by the lateral habenula ( $\mathrm{LHb}$ ), Quina et al injected an anterogradely transported viral vector encoding eGFP into the $\mathrm{LHb}$ under the regulation of a human synapsin promoter to define the anatomical position of the RMTg in mice by observing the presence and distribution of LHb input fibers within the RMTg. They found that the anatomical position of the RMTg extended from $3.64 \mathrm{~mm}$ to $4.60 \mathrm{~mm}$ posterior to the bregma. The RMTg of mice was found to extend to at least $4.50 \mathrm{~mm}$ posterior to the bregma and thereafter to be replaced by the anterior tegmental nucleus (ATg). ${ }^{18}$ In fact, the anatomical location of the RMTg is not overtly delineated on standard atlases, which do not distinguish this region from the adjacent $\mathrm{ATg}$, the pontine reticular nucleus nor the superior central nucleus raphe. Wasserman et al and Steidl et al performed experiments examining muscarinic control of RMTg GABAergic neurons in morphinemediated locomotion experiments, where the anatomical position of the RMTg in mice extended from $4.04 \mathrm{~mm}$ to $4.36 \mathrm{~mm}$ posterior to the bregma, ${ }^{19,20}$ which included only a part of the RMTg.

\section{Anatomical Location for RMTg Delimitation in Rats and Mice by Specific Molecular Markers}

Although c-Fos expression in response to aversive stimuli was initially used to define the RMTg, its exact expression pattern may vary with intensity and type of aversive stimulation. The method of observing LHb fibers may be more consistent, but there are also notable limitations due to the technical challenge of virus injection. Finally, although RMTg neurons are mainly GABAergic, such neurons are also densely distributed around the RMTg. Hence, there is a need to identify molecular markers more specifically expressed in this area, in order to distinguish the RMTg from adjacent brain structures and to precisely manipulate it.

In 2016, Lahti et al found that although RMTg GABAergic neurons have similar origins as VTA GABAergic neurons, they express different molecular biomarkers. VTA GABAergic neurons expressed Zfpm2 and Six3, while RMTg GABAergic neurons mainly expressed Sox2, FoxP1, and Sox14 during development stage. Furthermore, the transcription factor FoxP1 is also highly expressed in the adult RMTg in rats, suggesting that it could be used as a specific molecular marker to identify the RMTg. ${ }^{21}$ Smith et al further identified two specific molecular markers of the transcription factor FoxP1 and 
prepronociceptin (Pnoc) of RMTg neurons using RNA sequencing. Compared with Pnoc, FoxP1 is more specifically expressed in the RMTg. When the retrograde tracer CTB was injected into the VTA of rats, about $83 \%$ of CTB-positive neurons expressed FoxP1 within the RMTg region, but only $4.5 \%$ of CTB-labeled cells expressed FoxP1 outside the RMTg. Conversely, 55\% of CTB-labeled neurons in the RMTg expressed Pnoc. Similarly, in mice, the FoxP1positive neurons of the RMTg area were strongly colocalized with retrograde CTB, which was injected from the VTA. About $78 \%$ of CTB-positive neurons expressed FoxP1 in the RMTg versus $2 \%$ outside the RMTg. However, Pnoc could not be used to specifically identify the RMTg in mice since there is no particular higher expression of Pnoc within the RMTg. Instead, Pnoc is widely expressed in the surrounding area. ${ }^{22}$

A schematic diagram of the anatomical location of mouse RMTg is shown in Figure 1.

\section{Afferent and Efferent Connections of the RMTg Connections Among the RMTg and the $\mathrm{LHb}$ and Monoaminergic (MAergic) \\ Neurons}

The RMTg mainly receives innervation from the LHb, ${ }^{11,23}$ which projects into the midbrain and brainstem regions, riching in MAergic neurons. The RMTg sends a particularly dense innervation to DAergic neurons of the VTA and the SNc, and it also innervates the serotonin-rich dorsal raphe nucleus (DRN), albeit not necessarily the serotonin neurons themselves. ${ }^{24,25}$ There are direct as well as indirect projections of the $\mathrm{LHb}$ to MAergic cell groups. ${ }^{26-30}$ The glutamatergic (GAergic) neurons in the LHb directly innervate DAergic and GABAergic neurons in the VTA to a comparable extent. ${ }^{30}$ Similarly, neurons that directly innervate the DRN also come from the $\mathrm{LHb}^{31,32}$ The indirect innervation of the LHb on MAergic neurons is achieved through the relay station of the RMTg. The GAergic neurons in the lateral division of the $\mathrm{LHb}(\mathrm{LHbL})$ inhibit VTA DAergic neurons ${ }^{9-11}$ and GAergic neurons expressing vesicular glutamate transporter-3 (VGLUT3) in the $\mathrm{DRN}^{25,27,33}$ by indirect activation of RMTg GABAergic neurons. The VTA and SNc are the main downstream targets innervated by the RMTg and they also send minor projections back to the RMTg, suggesting that there are reciprocal projections between the RMTg and the midbrain dopamine system. ${ }^{11,34}$ Moreover, the input of the midbrain DAergic neurons is mediated by local non-DAergic neurons within or closely associated with the DAergic nuclei. The DAergic neurons and some of non-DAergic neurons also send functionally important outputs from the midbrain to forebrain targets. ${ }^{3,35}$

\section{Connections Between the RMTg and Other Nuclei}

In addition to receiving dense projections from the $\mathrm{LHb}$, the RMTg also gets strong inputs from structures with significant impacts on motor functions, such as deep layers of the superior colliculus (SC), cerebellar nuclei,
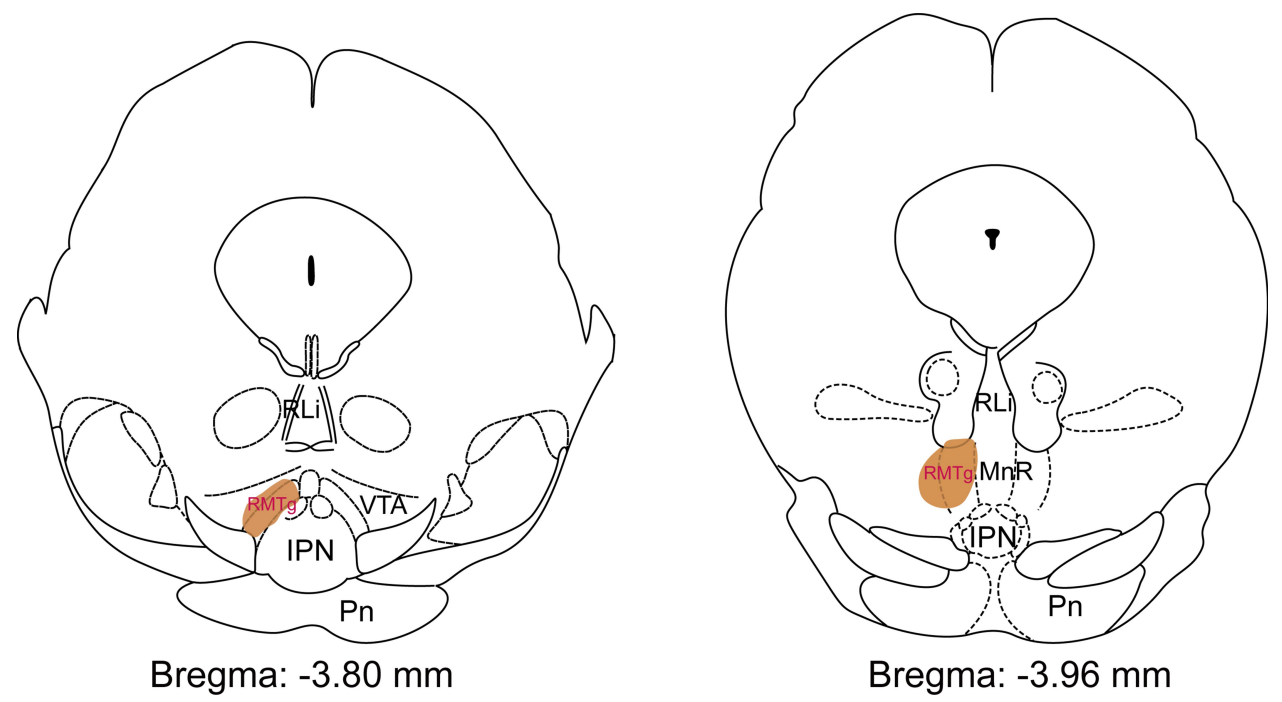

Figure I The location (shown as brown area) of the rostromedial tegmental nucleus (RMTg) mapped on coronal atlas by FoxPI staining in mice. The brown shading highlights the RMTg as shown in the Paxinos and Keith (200I) mouse brain atlas.

Abbreviations: RLi, rostral linear nucleus of the raphe; VTA, ventral tegmental area; IPN, interpeduncular nucleus; Pn, pontine nuclei; MnR, median raphe nucleus. 
retrorubral field, pontine reticular formation, dorsomedial tegmental area, and raphe interpositus. ${ }^{34}$ Tracing studies have also shown that the RMTg receives projections from multiple nuclei, including the shell of the nucleus accumbens (NAc), ventral pallidum (VP), bed nucleus of the stria terminalis (BNST), amygdala, lateral hypothalamus (LH), thalamic paraventricular nucleus, lateral septum (LS), lateral preoptic area (LPO), periaqueductal gray (PAG), interpeduncular nucleus, laterodorsal tegmental area (LDT), and some cortical neurons. ${ }^{9,11,36}$

The RMTg has robust inhibitory projections that contact preferentially DAergic neurons in the VTA, SNc, and retrorubral field. ${ }^{37,38}$ Furthermore, the RMTg also projects to the nuclei of the forebrain, midbrain, and brainstem, including the LH, LPO, NAc, BNST, LDT, DRN, PAG and pedunculopontine nucleus. ${ }^{36,39,40}$

\section{Functional Studies on the RMTg}

The RMTg is innervated by structures implicated in aversive emotional processes, including the LHb, amygdala, PAG, and LS. Moreover, there are strong projections from structures like the LH and SC implicated in body homeostasis, locomotion, and sensory integration. Furthermore, arousalassociated structures in the reticular formation and tegmental nuclei also send projections to the RMTg. Therefore, the RMTg may integrate a wide range of information concerning both the external environment and the internal state to modulate behavioral output. After the integration of various informative signals, the RMTg influences responses through its projections to DAergic systems in midbrain structures and other ascending aminergic systems in the forebrain to guide behaviors. ${ }^{38}$ The RMTg is mainly composed of GABAergic inhibitory neurons, which control the activities of DAergic neurons and also regulate the activities of MAergic neurons. Because monoamine systems play pivotal roles in motor and mood-related behaviors, a variety of neurological diseases are associated with the dysfunction of these neurotransmitters. As a result, the RMTg has been proposed to be a possible component of the emotional system and regulation of sleep-wake behavior.

\section{The RMTg and Sleep-Wake Behavior}

Severe sleep disorders have been observed in nearly all the patients with neuropsychiatric disorders such as depression, drug addiction, and Parkinson's disease, which are closely associated with DA dysfunction in the brain. ${ }^{41,42}$ Growing quantities of evidence suggest that dopaminecontaining neurons are important for maintenance of arousal in both humans and animals. ${ }^{43,44}$ Although the RMTg has been confirmed to inhibit the electrical activities of midbrain DAergic neurons, ${ }^{13,45}$ whether it is implicated in sleep-wake behavior was previously unknown.

Using a highly automated sleep-wake bioanalysis system and chemogenetics, Yang et al revealed for the first time that the RMTg not only promotes sleep, but also plays a vital role in maintaining sleep homeostasis. ${ }^{12}$ When RMTg neurons were activated, mainly GABAergic neurons, although this did not occur in a cell-type-specific manner, the rats showed a significant increase in amount of non-rapid eye movement (NREM) sleep. Electroencephalogram (EEG) power spectrum analysis revealed that the average slow-wave activity (SWA)-a commonly used quantitative measure of sleep intensity ${ }^{46}$ increased in the rats with activation of RMTg neurons. In order to investigate whether the RMTg plays a role in physiological sleep promotion, RMTg neurons were lesioned with the neurochemical toxin ibotenic acid. The rats with RMTg lesions showed a remarkable decrease in the amount of NREM sleep and lower SWA levels in NREM sleep during the first hour after lights on. To explore the role of the RMTg in homeostatic regulation of sleep, sleep deprivation (SD) was performed. ${ }^{47}$ During the subsequent sleep recovery period following 6-h forced arousal, the lesioned rats exhibited a similar increase in their NREM sleep amount as the control rats with intact RMTg neurons; however, 6-h SD did not induce an increase in SWA following SD in lesioned animals. These results clarify that the RMTg is an indispensable nucleus for NREM sleep initiation, maintenance, and the regulation of sleep homeostasis. This suggests that the RMTg is a promising therapeutic target for prolonging sleep and improving sleep quality for patients with sleep disorders.

Similarly, chemogenetic activation of GABAergic neurons in the anterior part of the VTA promoted NREM sleep, also in part by inhibition of the midbrain DAergic neurons. ${ }^{5,12}$ The difference of these two areas is that the activation of VTA GABAergic neurons primarily promoted NREM in dark period, while RMTg effects were mainly observed in light period. This fits with multiunit recordings from mouse VTA neurons, in which most cells fire more during the active phase. ${ }^{48}$ This indicates that GABAergic neurons of the VTA and the RMTg may fire differently from the circadian point of view.

\section{The RMTg and Substance Addictions}

In the diagnostic and statistical manual of mental disorders, fifth edition (DSM-5), substance use disorders, defined as disorders that are related to the taking of a drug of abuse (including alcohol), are essentially the 
amalgamation of substance dependence and substance abuse. $^{49}$ Addictive drugs, including morphine, heroin, nicotine, ethanol, as well as caffeine, may have both rewarding and aversive effects. ${ }^{50,51}$ In drug dependence, people lose control over the amount, frequency, and/or duration of drug intake, presumably for the pursuit of reward, such as euphoria, or alleviation of aversion, such as anxiety, during drug withdrawal. As drug abuse can be envisaged as a function of the balance between reward and aversion, the appetitive properties might result from direct activation of the neural reward system and/or inhibition of neural circuits mediating aversion. ${ }^{50,52}$ Studies have found that the RMTg is activated by aversive stimuli such as foot shock, fasting, or reward omission, demonstrated by c-Fos expression. ${ }^{10,53}$ The LHb is also activated by these stimuli, and it has been proposed to be one major contributor to these responses in the RMTg, although other sources also play important roles. ${ }^{54}$ Following aversive stimuli, the $\mathrm{LHb}$ receives signal inputs from the limbic system and basal ganglia, integrates them, and sends projections to the RMTg, which inhibits reward-encoding midbrain DAergic neurons by releasing GABA. ${ }^{52}$ It has been reported that the activation of the RMTg can enhance the extinction retention of the extinction learning of cocaine selfadministration in rats, while the RMTg inactivation before a cue-induced reinstatement session or inactivation alone before an extinction session increased overall lever pressing. 55

The $\mu$-opioid receptor is the main in vivo molecular target for opiates. It was initially believed that opiates activate VTA DAergic neurons via inhibiting local GABAergic interneurons within the VTA. ${ }^{5,57}$ However, recent work showed that $\mu$-opioid receptors are more strongly expressed in the RMTg than in the VTA in rats. Systemic administration of opiates, such as morphine, was found to strongly inhibit the spontaneous firing rate of RMTg neurons in vivo. The excitatory postsynaptic current amplitude evoked by stimulation of rostral afferents was significantly reduced by bath application of morphine. $^{52}$ Opiates increase the firing rate of VTA DAergic neurons by acting on $\mu$-opioid receptors on RMTg neurons and their terminals inside the VTA, whereas intra-VTA delivery of morphine cannot increase dopamine cell activity if the RMTg has been silenced by muscimol, a $\mathrm{GABA}_{\mathrm{A}}$ receptor agonist. ${ }^{58,59}$ These results indicate that the RMTg is a primary target of opioids, which might contribute to their rewarding properties by disinhibiting the release of DA within the VTA. ${ }^{60}$
Alcohol dependence is a serious health problem that worsens over time. Loss of alcohol aversion is an important cause for alcohol dependence and relapse after withdrawal. For example, heavy drinkers often reported experiencing greater reward and stimulating responses and fewer aversive effects of alcohol than light drinkers. ${ }^{61}$ Ethanol consumption was significantly decreased upon activation of the RMTg by infusion of alpha-amino-3-hydroxy-5-methyl-4isoxazolepropionic acid (AMPA), but was increased upon inhibition of the RMTg and the alcohol-withdrawalinduced anxiety-like behavior was reversed. ${ }^{62-65}$

In a conditioned taste aversion (CTA) experiment in rats where exposure to a saccharin solution was paired with intraperitoneal administration of ethanol, the expression of c-Fos was significantly increased in both the RMTg and the $\mathrm{LHb}$, which was positively correlated with CTA magnitude. $^{66}$ When dermorphin-saporin, a neurotoxin, was employed to lesion RMTg neurons, rats' ethanol intake and self-administration as well as preference for ethanol substantially increased. These results suggest that ethanol is less aversive to rats with RMTg destruction; thus, the RMTg contributes significantly to the regulation of ethanol consumption. ${ }^{67}$ The NAc is a novel brain region for treatment of alcohol dependence by modulation of DAergic pathways. ${ }^{68,69}$ The RMTg may be involved in the circuits regulating alcohol addiction by modulation of dopamine release in the NAc because the RMTg is recognized as a brake of the dopamine system. ${ }^{10}$

The VP, a primary output of the NAc, projects to the VTA and the LHb and also plays a critical role in processing of aversive stimuli. Tooley et al observed that optogenetic activation of GAergic VP neurons increased the firing rate of $\mathrm{LHb}, \mathrm{RMTg}$, and VTA GABAergic neurons and induced place avoidance in mice. In contrast, viral ablation of these neurons increased reward response and abolished taste aversion to sucrose. This suggests that the activation of VP GAergic neurons could inhibit the activities of VTA DAergic neurons via activating VTA or RMTg GABAergic neurons and therefore reduce the release of dopamine as a result of producing an addictionassociated aversive behavior. ${ }^{70}$ Furthermore, a recent study has shown that there are three distinct afferents to the RMTg from prelimbic cortex, brainstem parabrachial nucleus, and $\mathrm{LHb}$ that drive triply dissociable RMTg responses to aversive cues, outcomes and prediction errors, respectively. ${ }^{54}$ As for the downstream, data show that RMTg neurons broadcast punishment signals to multiple targets including the VTA and SNc. ${ }^{53,54,71}$ 
Overall, the results of the existing studies suggest that the activities of RMTg neurons are profoundly influenced by drugs of abuse and, as important inhibitory afferents to midbrain DAergic neurons, they might play important roles in the complex interplay between the neural circuits mediating aversion and reward.

\section{Differences Between the LHb and the RMTg}

Although the RMTg and the LHb both regulate various behavioral responses to aversive stimuli, including unpredictable footshock, there are differences between these two parts of the brain. ${ }^{72}$ Instead of serving as an aversion or anti-reward center, the LHb integrates rewarding and aversive signals for expression of preferences. For example, inactivation of the LHb abolished choice bias, ${ }^{73}$ but no such evidence has shown that the RMTg combines opposite signals to integrate information. The LHb contains not only a large number of GAergic neurons but also GABAergic neurons. Recently, Flanigan et al showed that the activation of GABAergic neurons in the $\mathrm{LHb}$ promoted male-male aggression and these GABAergic neurons were inhibitory within the LHb and dampened the activity of the LHb overall. ${ }^{74}$ These data suggest that there are local circuits within the LHb. There is consensus that most of the neurons in the RMTg are GABAergic. Whether there are any local circuits within the nucleus has yet to be determined. Currently, some evidence shows that the LHb is a component of the extended neural circadian system. It receives innervation from the suprachiasmatic nuclei and the intrinsically photosensitive retinal ganglion cells. Daily changes in molecular clock activity of Perl, Per2, and Bmall were reported. ${ }^{75}$ Whether circadian information in the $\mathrm{LHb}$ regulates functions through downstream targets such as the RMTg needs to be investigated.

\section{Conclusion}

Current studies have confirmed that the RMTg acts as a major inhibitory nucleus in the midbrain. It integrates multimodal information inputs and modulates activities of its descending nuclei to modulate sleep-wake, addiction, and other motivation-related behaviors. According to the existing literature, including our own studies, the majority of neurons in the RMTg are GABAergic neurons. Recent studies using RNA sequencing showed that the transcription factor FoxP1 is specifically expressed at high levels in the mouse RMTg. This suggests that in addition to vesicular GABA transporter (VGAT)-Cre and GAD2-Cre mice, the development of FoxP1-Cre mice will provide even greater selectivity for targeting the RMTg in future research. Additionally, the results of distinct afferents of the RMTg driving dissociable responses indicate that the RMTg may include topographically distinct subpopulations with different functions through special output targets.

Future studies will investigate the possible roles and neural mechanisms of the RMTg in the development of psychiatric and neurological disorders, such as sleep disorders, substance use disorders, depression, and Parkinson's disease. These findings will inform the development of more efficient therapeutic approaches to mental illness.

\section{Acknowledgment}

The authors thank the National Natural Science Foundation of China (grant number 81571296, 8202010801, 32070984, 31530035) for support.

\section{Author Contributions}

All authors contributed to data analysis, drafting or revising the article, have agreed on the journal to which the article will be submitted, gave final approval of the version to be published, and agree to be accountable for all aspects of the work.

\section{Disclosure}

The authors declare that they have no conflicts of interest.

\section{References}

1. Swanson LW. The projections of the ventral tegmental area and adjacent regions: a combined fluorescent retrograde tracer and immunofluorescence study in the rat. Brain Res Bull. 1982;9(1-6):321-353. doi:10.1016/0361-9230(82)90145-9

2. Dobi A, Margolis EB, Wang HL, Harvey BK, Morales M. Glutamatergic and nonglutamatergic neurons of the ventral tegmental area establish local synaptic contacts with dopaminergic and nondopaminergic neurons. J Neurosci. 2010;30(1):218-229. doi:10.1523/ JNEUROSCI.3884-09.2010

3. Taylor SR, Badurek S, Dileone RJ, Nashmi R, Minichiello L, Picciotto MR. GABAergic and glutamatergic efferents of the mouse ventral tegmental area. J Comp Neurol. 2014;522(14):3308-3334. doi: $10.1002 /$ cne. 23603

4. Yu X, Ba W, Zhao G, et al. Dysfunction of ventral tegmental area GABA neurons causes mania-like behavior. Mol Psychiatry. 2020. doi:10.1038/s41380-020-0810-9

5. Yu X, Li W, Ma Y, et al. GABA and glutamate neurons in the VTA regulate sleep and wakefulness. Nat Neurosci. 2019;22(1):106-119. doi:10.1038/s41593-018-0288-9

6. Zhou Z, Liu X, Chen S, et al. A VTA GABAergic neural circuit mediates visually evoked innate defensive responses. Neuron. 2019;103(3):473-488. doi:10.1016/j.neuron.2019.05.027 
7. Perrotti LI, Bolaños CA, Choi $\mathrm{K}$, et al. $\triangle \mathrm{FosB}$ accumulates in a GABAergic cell population in the posterior tail of the ventral tegmental area after psychostimulant treatment. Eur J Neurosci. 2005;21(10):2817-2824.

8. Jhou T. Neural mechanisms of freezing and passive aversive behaviors. J Comp Neurol. 2005;493(1):111-114. doi:10.1002/ cne. 20734

9. Kaufling J, Veinante P, Pawlowski SA, Freund-Mercier M, Barrot M. Afferents to the GABAergic tail of the ventral tegmental area in the rat. J Comp Neurol. 2009;513(6):597-621. doi:10.1002/cne.21983

10. Jhou TC, Fields HL, Baxter MG, Saper CB, Holland PC. The rostromedial tegmental nucleus (RMTg), a GABAergic afferent to midbrain dopamine neurons, encodes aversive stimuli and inhibits motor responses. Neuron. 2009;61(5):786-800. doi:10.1016/j.neuron.2009.02.001

11. Jhou TC, Geisler S, Marinelli M, Degarmo BA, Zahm DS. The mesopontine rostromedial tegmental nucleus: a structure targeted by the lateral habenula that projects to the ventral tegmental area of Tsai and substantia nigra compacta. $J$ Comp Neurol. 2009;513 (6):566-596. doi:10.1002/cne.21891

12. Yang $\mathrm{S}, \mathrm{Hu} \mathrm{Z}$, Luo $\mathrm{Y}$, et al. The rostromedial tegmental nucleus is essential for non-rapid eye movement sleep. PLoS Biol. 2018;16(4): e2002909. doi:10.1371/journal.pbio.2002909

13. Bourdy R, Sanchez-Catalan MJ, Kaufling J, et al. Control of the nigrostriatal dopamine neuron activity and motor function by the tail of the ventral tegmental area. Neuropsychopharmacol. 2014;39 (12):2788-2798. doi:10.1038/npp.2014.129

14. Ferreira JG, Del-Fava F, Hasue RH, Shammah-Lagnado SJ. Organization of ventral tegmental area projections to the ventral tegmental area-nigral complex in the rat. Neuroscience. 2008;153 (1):196-213. doi:10.1016/j.neuroscience.2008.02.003

15. Scammell TE, Estabrooke IV, McCarthy MT, et al. Hypothalamic arousal regions are activated during modafinil-induced wakefulness J Neurosci. 2000;20(22):8620-8628. doi:10.1523/JNEUROSCI.2022-08620.2000

16. Colussi-Mas J, Geisler S, Zimmer L, Zahm DS, Berod A. Activation of afferents to the ventral tegmental area in response to acute amphetamine: a double-labelling study. Eur $J$ Neurosci. 2007;26 (4):1011-1025. doi:10.1111/j.1460-9568.2007.05738.x

17. Geisler S, Marinelli M, Degarmo B, et al. Prominent activation of brainstem and pallidal afferents of the ventral tegmental area by cocaine Neuropsychopharmacol. 2008;33(11):2688-2700. doi:10.1038/sj.npp. 1301650

18. Quina LA, Tempest L, Ng L, et al. Efferent pathways of the mouse lateral habenula. J Comp Neurol. 2015;523(1):32-60. doi:10.1002/ cne. 23662

19. Wasserman DI, Tan JM, Kim JC, Yeomans JS. Muscarinic control of rostromedial tegmental nucleus GABA neurons and morphine-induced locomotion. Eur J Neurosci. 2016;44(1):1761-1770. doi:10.1111/ ejn. 13237

20. Steidl S, Dhillon ES, Sharma N, Ludwig J. Muscarinic cholinergic receptor antagonists in the VTA and RMTg have opposite effects on morphine-induced locomotion in mice. Behav Brain Res. 2017;323:111-116. doi:10.1016/j.bbr.2017.01.039

21. Lahti L, Haugas M, Tikker L, et al. Differentiation and molecular heterogeneity of inhibitory and excitatory neurons associated with midbrain dopaminergic nuclei. Development. 2016;143(3):516-529. doi:10.1242/dev.129957

22. Smith RJ, Vento PJ, Chao YS, Good CH, Jhou TC. Gene expression and neurochemical characterization of the rostromedial tegmental nucleus (RMTg) in rats and mice. Brain Struct Funct. 2018;10-1007.

23. Herkenham M, Nauta WJ. Efferent connections of the habenular nuclei in the rat. J Comp Neurol. 1979;187(1):19-47. doi:10.1002/ cne. 901870103

24. Metzger M, Souza R, Lima LB, et al. Habenular connections with the dopaminergic and serotonergic system and their role in stress-related psychiatric disorders. Eur J Neurosci. 2019. doi:10.1111/ejn.14647
25. Sego C, Goncalves L, Lima L, Furigo IC, Donato JJ, Metzger M. Lateral habenula and the rostromedial tegmental nucleus innervate neurochemically distinct subdivisions of the dorsal raphe nucleus in the rat. J Comp Neurol. 2014;522(7):1454-1484. doi:10.1002/cne.23533

26. Goncalves L, Sego C, Metzger M. Differential projections from the lateral habenula to the rostromedial tegmental nucleus and ventral tegmental area in the rat. $J$ Comp Neurol. 2012;520(6):1278-1300. doi:10.1002/cne. 22787

27. Metzger M, Bueno D, Lima LB. The lateral habenula and the serotonergic system. Pharmacol Biochem Behav. 2017;162:22-28. doi:10.1016/j.pbb.2017.05.007

28. Petzel A, Bernard R, Poller WC, Veh RW. Anterior and posterior parts of the rat ventral tegmental area and the rostromedial tegmental nucleus receive topographically distinct afferents from the lateral habenular complex. J Comp Neurol. 2017;525(10):2310-2327. doi:10.1002/cne. 24200

29. Brown PL, Palacorolla H, Brady D, Riegger K, Elmer GI, Shepard PD. Habenula-induced inhibition of midbrain dopamine neurons is diminished by lesions of the rostromedial tegmental nucleus. $J$ Neurosci. 2017;37(1):217-225. doi:10.1523/JNEUROSCI.1353-16.2016

30. Omelchenko N, Bell R, Sesack SR. Lateral habenula projections to dopamine and GABA neurons in the rat ventral tegmental area. Eur $J$ Neurosci. 2009;30(7):1239-1250. doi:10.1111/j.1460-9568.2009. 06924.x

31. Bernard R, Veh RW. Individual neurons in the rat lateral habenular complex project mostly to the dopaminergic ventral tegmental area or to the serotonergic raphe nuclei. J Comp Neurol. 2012;520 (11):2545-2558. doi:10.1002/cne. 23080

32. Kim U. Topographic commissural and descending projections of the habenula in the rat. $J$ Comp Neurol. 2009;513(2):173-187. doi:10.1002/cne.21951

33. Gras C, Herzog E, Bellenchi GC, et al. A third vesicular glutamate transporter expressed by cholinergic and serotoninergic neurons. J Neurosci. 2002;22(13):5442-5451. doi:10.1523/JNEUROSCI.2213-05442.2002

34. Yetnikoff L, Cheng AY, Lavezzi HN, Parsley KP, Zahm DS. Sources of input to the rostromedial tegmental nucleus, ventral tegmental area, and lateral habenula compared: a study in rat. J Comp Neurol. 2015;523(16):2426-2456. doi:10.1002/cne.23797

35. Morello F, Partanen J. Diversity and development of local inhibitory and excitatory neurons associated with dopaminergic nuclei. FEBS Lett. 2015;589(24Pt A):3693-3701. doi:10.1016/j.febslet.2015.10.001

36. Fakhoury M. The tail of the ventral tegmental area in behavioral processes and in the effect of psychostimulants and drugs of abuse. Prog Neuropsychopharmacol Biol Psychiatry. 2018;84(Pt A):30-38. doi:10.1016/j.pnpbp.2018.02.002

37. Barrot M, Sesack SR, Georges F, Pistis M, Hong S, Jhou TC. Braking dopamine systems: a new GABA master structure for mesolimbic and nigrostriatal functions. J Neurosci. 2012;32(41):14094-14101. doi:10.1523/JNEUROSCI.3370-12.2012

38. Bourdy R, Barrot M. A new control center for dopaminergic systems: pulling the VTA by the tail. Trends Neurosci. 2012;35(11):681-690. doi:10.1016/j.tins.2012.06.007

39. Kaufling J, Veinante P, Pawlowski SA, Freund-Mercier M, Barrot M. $\gamma$-Aminobutyric acid cells with cocaine-induced $\Delta$ FosB in the ventral tegmental area innervate mesolimbic neurons. Biol Psychiatry. 2010;67(1):88-92. doi:10.1016/j.biopsych.2009.08.001

40. Lavezzi HN, Parsley KP, Zahm DS. Mesopontine rostromedial tegmental nucleus neurons projecting to the dorsal raphe and pedunculopontine tegmental nucleus: psychostimulant-elicited Fos expression and collateralization. Brain Struct Funct. 2012;217(3):719-734. doi:10.1007/s00429-011-0368-z

41. Irwin MR, Bjurstrom MF, Olmstead R. Polysomnographic measures of sleep in cocaine dependence and alcohol dependence: implications for age-related loss of slow wave, stage 3 sleep. Addiction. 2016;111 (6):1084-1092. doi:10.1111/add.13300 
42. Louter M, Aarden WC, Lion J, Bloem BR, Overeem S. Recognition and diagnosis of sleep disorders in Parkinson's disease. $J$ Neurol. 2012;259(10):2031-2040. doi:10.1007/s00415-012-6505-7

43. Eban-Rothschild A, Rothschild G, Giardino WJ, Jones JR, de Lecea L. VTA dopaminergic neurons regulate ethologically relevant sleep-wake behaviors. Nat Neurosci. 2016;19(10):1356-1366. doi:10.1038/nn.4377

44. Saper CB, Fuller PM, Pedersen NP, Lu J, Scammell TE. Sleep state switching. Neuron. 2010;68(6):1023-1042. doi:10.1016/j.neuron. 2010.11.032

45. Balcita-Pedicino JJ, Omelchenko N, Bell R, Sesack SR. The inhibitory influence of the lateral habenula on midbrain dopamine cells: ultrastructural evidence for indirect mediation via the rostromedial mesopontine tegmental nucleus. J Comp Neurol. 2011;519 (6):1143-1164. doi:10.1002/cne.22561

46. Deboer T. Behavioral and electrophysiological correlates of sleep and sleep homeostasis. Curr Top Behav Neurosci. 2015;25:1-24.

47. Yang SR, Sun H, Huang ZL, Yao MH, Qu WM. Repeated sleep restriction in adolescent rats altered sleep patterns and impaired spatial learning/memory ability. Sleep. 2012;35(6):849-859. doi: $10.5665 /$ sleep. 1888

48. Fifel K, Meijer JH, Deboer T. Circadian and homeostatic modulation of multi-unit activity in midbrain dopaminergic structures. Sci Rep. 2018;8(1):7765. doi:10.1038/s41598-018-25770-5

49. American Psychiatric Association. Diagnostic and Statistical Manual of Mental Disorders. 5th ed. Washington, DC: American Psychiatric Association; 2013.

50. Verendeev A, Riley AL. The role of the aversive effects of drugs in self-administration: assessing the balance of reward and aversion in drug-taking behavior. Behav Pharmacol. 2013;24(5-6):363-374. doi:10.1097/FBP.0b013e32836413d5

51. Grant JE, Chamberlain SR. Expanding the definition of addiction: DSM-5 vs. ICD-11. CNS Spectr. 2016;21(4):300-303. doi:10.1017/ S1092852916000183

52. Lecca S, Melis M, Luchicchi A, et al. Effects of drugs of abuse on putative rostromedial tegmental neurons, inhibitory afferents to midbrain dopamine cells. Neuropsychopharmacol. 2011;36(3):589-602. doi:10.1038/npp.2010.190

53. Li H, Pullmann D, Cho JY, Eid M, Jhou TC. Generality and opponency of rostromedial tegmental (RMTg) roles in valence processing. Elife. 2019;10-7554.

54. Li H, Vento PJ, Parrilla-Carrero J, et al. Three rostromedial tegmental afferents drive triply dissociable aspects of punishment learning and aversive valence encoding. Neuron. 2019;104(5):987-999. doi:10. 1016/j.neuron.2019.08.040

55. Huff ML, LaLumiere RT. The rostromedial tegmental nucleus modulates behavioral inhibition following cocaine self-administration in rats. Neuropsychopharmacol. 2015;40(4):861-873. doi:10.1038/npp.2014.260

56. Johnson SW, North RA. Opioids excite dopamine neurons by hyperpolarization of local interneurons. $J$ Neurosci. 1992;12(2):483-488. doi:10.1523/JNEUROSCI.12-02-00483.1992

57. Lecca S, Melis M, Luchicchi A, Muntoni AL, Pistis M. Inhibitory inputs from rostromedial tegmental neurons regulate spontaneous activity of midbrain dopamine cells and their responses to drugs of abuse. Neuropsychopharmacol. 2012;37(5):1164-1176. doi:10.1038/ npp. 2011.302

58. Jalabert M, Bourdy R, Courtin J, et al. Neuronal circuits underlying acute morphine action on dopamine neurons. Proc Natl Acad Sci. 2011;108(39):16446-16450. doi:10.1073/pnas.1105418108

59. Matsui A, Williams JT. Opioid-sensitive GABA inputs from rostromedial tegmental nucleus synapse onto midbrain dopamine neurons. $J$ Neurosci. 2011;31(48):17729-17735. doi:10.1523/JNEUROSCI. 4570-11.2011
60. Barrot M. Ineffective VTA disinhibition in protracted opiate withdrawal. Trends Neurosci. 2015;38(11):672-673. doi:10.1016/j. tins.2015.08.004

61. Gilpin NW, Koob GF. Neurobiology of alcohol dependence: focus on motivational mechanisms. Alcohol Res Health. 2008;31(3):185-195.

62. Fu R, Zuo W, Gregor D, Li J, Grech D, Ye JH. Pharmacological manipulation of the rostromedial tegmental nucleus changes voluntary and operant ethanol self-administration in rats. Alcohol Clin Exp Res. 2016;40(3):572-582. doi:10.1111/acer.12974

63. Glover EJ, Starr EM, Chao Y, Jhou TC, Chandler LJ. Inhibition of the rostromedial tegmental nucleus reverses alcohol withdrawal-induced anxiety-like behavior. Neuropsychopharmacol. 2019;44(11):1896-1905. doi:10.1038/s41386-019-0406-8

64. Melis M, Sagheddu C, De Felice M, et al. Enhanced endocannabinoid-mediated modulation of rostromedial tegmental nucleus drive onto dopamine neurons in sardinian alcohol-preferring rats. J Neurosci. 2014;34(38):12716-12724. doi:10.1523/JNEUROSCI.184414.2014

65. Sheth C, Furlong TM, Keefe KA, Taha SA. Lesion of the rostromedial tegmental nucleus increases voluntary ethanol consumption and accelerates extinction of ethanol-induced conditioned taste aversion. Psychopharmacology (Berl). 2016;233(21-22):3737-3749. doi:10.1007/s00213-016-4406-7

66. Glover EJ, McDougle MJ, Siegel GS, Jhou TC, Chandler LJ. Role for the rostromedial tegmental nucleus in signaling the aversive properties of alcohol. Alcohol Clin Exp Res. 2016;40(8):1651-1661. doi:10.1111/acer.13140

67. Fu R, Chen $\mathrm{X}$, Zuo $\mathrm{W}$, et al. Ablation of mu opioid receptor-expressing GABA neurons in rostromedial tegmental nucleus increases ethanol consumption and regulates ethanol-related behaviors. Neuropharmacology. 2016;107:58-67. doi:10.1016/j. neuropharm.2016.02.027

68. Cannizzaro C, Talani G, Brancato A, et al. Dopamine restores limbic memory loss, dendritic spine structure, and NMDAR-dependent LTD in the nucleus accumbens of alcohol-withdrawn rats. J Neurosci. 2019;39(5):929-943. doi:10.1523/JNEUROSCI.1377-18.2018

69. Vannemreddy P, Slavin K. Nucleus accumbens as a novel target for deep brain stimulation in the treatment of addiction: a hypothesis on the neurochemical and morphological basis. Neurol India. 2019;67 (5):1220-1224. doi:10.4103/0028-3886.271239

70. Tooley J, Marconi L, Alipio JB, et al. Glutamatergic ventral pallidal neurons modulate activity of the habenula-tegmental circuitry and constrain reward seeking. Biol Psychiatry. 2018;83(12):1012-1023. doi:10.1016/j.biopsych.2018.01.003

71. Sun Y, Cao J, Xu C, Liu X, Wang Z, Zhao H. Rostromedial tegmental nucleus-substantia nigra pars compacta circuit mediates aversive and despair behavior in mice. Exp Neurol. 2020;333:113433. doi:10.1016/j.expneurol.2020.113433

72. Jhou TC, Vento PJ. Bidirectional regulation of reward, punishment, and arousal by dopamine, the lateral habenula and the rostromedial tegmentum (RMTg). Curr Opin Behav Sci. 2019;26:90-96. doi:10.1016/j.cobeha.2018.11.001

73. Stopper CM, Floresco SB. What's better for me? Fundamental role for lateral habenula in promoting subjective decision biases. Nat Neurosci. 2014;17(1):33-35. doi:10.1038/nn.3587

74. Flanigan ME, Aleyasin $\mathrm{H}$, Li L, et al. Orexin signaling in GABAergic lateral habenula neurons modulates aggressive behavior in male mice. Nat Neurosci. 2020;23(5):638-650. doi:10.1038/ s41593-020-0617-7

75. Bano-Otalora B, Piggins HD. Contributions of the lateral habenula to circadian timekeeping. Pharmacol Biochem Behav. 2017;162:46-54. 
Nature and Science of Sleep

Dovepress

\section{Publish your work in this journal}

Nature and Science of Sleep is an international, peer-reviewed, open access journal covering all aspects of sleep science and sleep medicine, including the neurophysiology and functions of sleep, the genetics of sleep, sleep and society, biological rhythms, dreaming, sleep disorders and therapy, and strategies to optimize healthy sleep.

Submit your manuscript here: https://www.dovepress.com/nature-and-science-of-sleep-journa
The manuscript management system is completely online and includes a very quick and fair peer-review system, which is all easy to use. Visit http://www.dovepress.com/testimonials.php to read real quotes from published authors. 